\title{
Vorfälligkeitsentschädigung und steuerliche Abzugsfähigkeit
}

Thomas J. Wenger *

Wer eine Festhypothek vor Ablauf der festen Laufzeit auflöst, muss in der Regel eine sogenannte Vorfälligkeitsentschädigung bezahlen. Das Bundesgericht befasst sich nun in zwei neuen Urteilen mit der Frage, wie solche Zahlungen bei der Einkommenssteuer bzw. bei der Grundstückgewinnsteuer zu berücksichtigen sind. Die beiden Entscheide stehen im Widerspruch zu einzelnen Lehrmeinungen und kantonalen Steuerpraktiken. Sie werden daher zu einer Harmonisierung führen, bringen aber Abgrenzungsfragen mit sich und können bei pendenten Fällen zu unbefriedigenden Veranlagungsentscheiden führen.

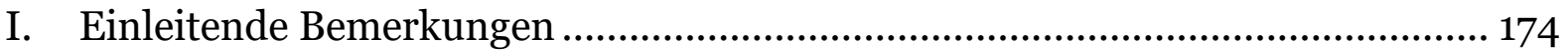

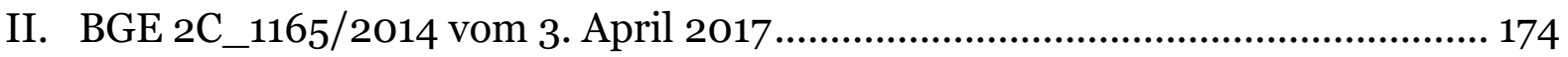

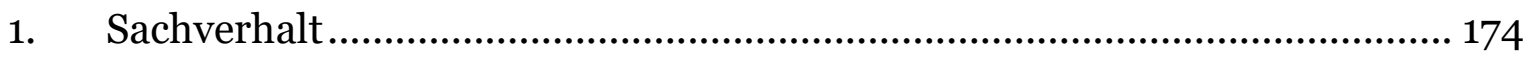

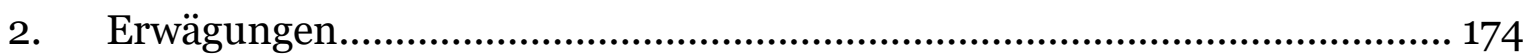

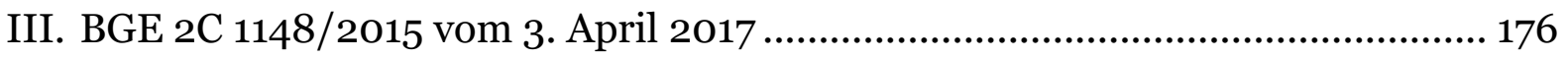

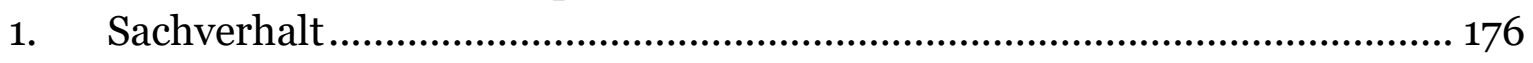

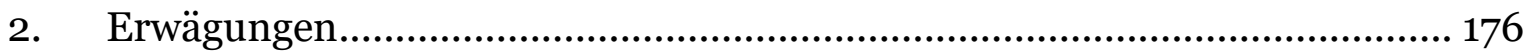

IV. Fazit der beiden Entscheide ............................................................... 178

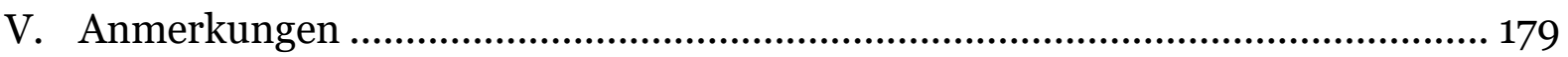

1. Vorbemerkungen.............................................................................. 179

2. Bisherige kantonale Praxis................................................................ 179

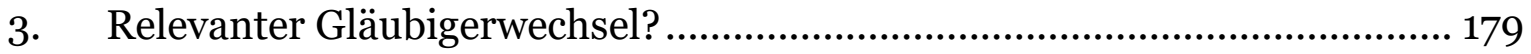

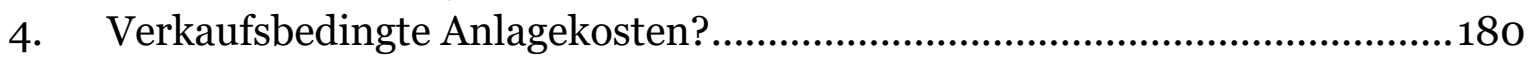

5. Rechtliche Wertvermehrung? .............................................................. 180

6. Rechtsnatur der Vorfälligkeitsentschädigung ........................................ 181

7. Besteuerung nach der wirtschaftlichen Leistungsfähigkeit ........................ 182

8. Praktische Herausforderungen .............................................................. 183

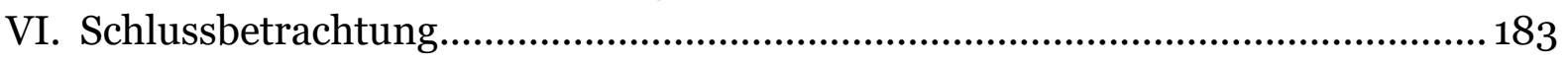

Zitiervorschlag: Thomas J. Wenger, Vorfälligkeitsentschädigung und steuerliche Abzugsfähigkeit, in: sui-generis 2017, S. 173

URL: $\quad$ sui-generis.ch/42

DOI: $\quad$ https://doi.org/10.21257/sg.42

* Thomas J. Wenger, lic. iur, Notar, ist Partner bei Häusermann + Partner, Bern.

Der Autor dankt Frau Michelle Oswald, MLaw, Notariatspraktikantin bei Häusermann + Partner, Bern, für die wertvolle Unterstützung bei der Recherche zu diesem Beitrag, sowie Herrn Errol M. Küffer, lic. iur, Notar in Ittigen, für die kritische Durchsicht des Manuskripts.

Dieses Werk ist lizenziert unter einer Creative Commons Namensnennung - Weitergabe unter gleichen Bedingungen 4.0 International Lizenz. 


\section{Einleitende Bemerkungen}

1 Hypotheken treten in der Praxis oft als sogenannte «Festhypothek» auf, bei welcher der Zinssatz für eine feste Laufzeit fixiert wird. Unerwartete Gründe wie der Verkauf der Immobilie, Tod, Invalidität oder Scheidung können dazu führen, dass eine vorzeitige Auflösung notwendig wird. Der Kreditnehmer hat seinem Kreditgeber diesfalls meist eine sogenannte Vorfälligkeitsentschädigung (auch Abstandszahlung, Rücktrittsprämie, Ausstiegsentschädigung, Ablösesumme oder Penalty genannt) zu entrichten. Diese soll den Zinsmargenverlust des Kreditgebers ausgleichen, der bis zum ordentlichen Ablauf noch hätte erwirtschaftet werden können.

Steigende Immobilienpreise und das historisch tiefe Zinsniveau haben dazu beigetragen, dass sich in den letzten Jahren vermehrt Fragen zur steuerlichen Berücksichtigung von Vorfälligkeitsentschädigungen stellen. Es überrascht daher nicht, dass sich das Bundesgericht nun gleich in zwei Leiturteilen mit den Voraussetzungen für deren steuerliche Abzugsfähigkeit befasst. Konkret stellen sich folgende Fragen:

- Handelt es sich bei Vorfälligkeitsentschädigungen um Schuldzinsen, die bei der Einkommenssteuer abgezogen werden können?

- Können Vorfälligkeitsentschädigungen bei der Grundstückgewinnsteuer als Anlagekosten abgezogen werden, wenn sie im Zusammenhang mit dem Verkauf der Liegenschaft anfallen?

\section{BGE 2C_1165/2014 vom 3. April 2017}

\section{Sachverhalt}

3 Eine Wohnung im Kanton Neuenburg sollte ohne hypothekarische Belastung verkauft werden. Die Verkäufer lösten die bestehende Festhypothek daher gegen Bezahlung einer Vorfälligkeitsentschädigung und einer Bearbeitungsgebühr auf.

4 In der Steuererklärung wurden diese Entschädigungen bei der Einkommenssteuer in Abzug gebracht. Die Neuenburger Steuerbehörde hat den Abzug nicht gewährt. Die dagegen gerichtete Einsprache blieb ebenso erfolglos wie die anschliessende Beschwerde an das Kantonsgericht.

5 Mit Beschwerde in öffentlich-rechtlichen Angelegenheiten haben die Verkäufer vor Bundesgericht beantragt, das verwaltungsgerichtliche Urteil aufzuheben. Die Vorfälligkeitsentschädigung sei als Schuldzins zum Abzug zuzulassen. Nicht Gegenstand des Verfahrens war die Frage der Anrechenbarkeit bei der Grundstückgewinnsteuer.

\section{Erwägungen}

6 Gemäss Art. 33 Abs. 1 lit. a DBG ${ }^{1}$ können private Schuldzinsen von den Einkünften im Umfang der steuerbaren Vermögenserträge und weiterer 50'ooo.- Franken abgezogen werden.

7 Der Begriff «Schuldzins» ist wirtschaftlich zu betrachten. Es handelt sich dabei um ein Entgelt für die Gewährung eines Kapitals, sofern dieses Entgelt nach der

Bundesgesetz vom 14. Dezember 1990 über die direkte Bundessteuer (DBG; SR 642.11). 
Zeit und als Quote des Kapitals regelmässig in Prozenten berechnet wird. Das Bestehen einer Kapitalschuld ist daher begriffsnotwendig. ${ }^{2}$

Das Bundesgericht verweist auf die unterschiedlichen kantonalen Praktiken. In den Kantonen Basel-Landschaft, Zürich und Waadt können Vorfälligkeitsentschädigungen beim Einkommen in Abzug gebracht werden, während sie in den Kantonen Tessin, Luzern und Neuenburg nicht als Schuldzinsen im Sinne von Art. 33 Abs. 1 lit. a DBG anerkannt werden. 3

9 Eine Qualifikation der Vorfälligkeitsentschädigungen als Schuldzinsen ist dann ausgeschlossen, wenn es sich um eine Konventionalstrafe gemäss Art. $160 \mathrm{ff}$. OR4 handelt. Anders zu beurteilen ist dagegen eine Entschädigung, die in Abhängigkeit von der Zinssatzdifferenz und der Restlaufzeit des Darlehensvertrags ermittelt wird. Diesfalls überwiegt die Zinskomponente, so dass sich ein Abzug gemäss Art. 33 Abs. 1 lit. a DBG unter gewissen Umständen rechtfertigt.5 Ein solcher einkommenssteuerrelevanter Abzug ist dann angebracht, wenn das ursprüngliche Darlehensverhältnis zwischen dem Eigentümer und dem Kreditinstitut nicht endet, sondern nur dessen Konditionen verändert werden, z.B. wenn der Zinssatz oder die Kredithöhe umgestaltet werden oder wenn ein Wechsel zu einem anderen Hypothekarmodell beim gleichen Kredit- geber erfolgt. ${ }^{6}$ Bei einer solchen Konstellation findet - betrachtet man das Kreditverhältnis als Ganzes und nicht bloss die einzelne Hypothek - eine blosse Umschuldung statt. Zwischen der Vorfälligkeitsentschädigung und dem Schuldverhältnis besteht eine Verbindung, die so eng ist, dass sich eine Behandlung der Zahlung als Schuldzins - anstatt als Schadenersatz oder Konventionalstrafe rechtfertigt. 7

10 In der anderen - und im konkreten Fall $\mathrm{zu}$ beurteilenden - Konstellation erfolgt die vorzeitige Ablösung der Hypothek im Hinblick auf den Verkauf der Liegenschaft. Diesfalls findet eine vollständige Vertragsaufhebung statt und nicht bloss eine Umschuldung. Weder führt der Verkäufer mit dem bisherigen Gläubiger das Hypothekarverhältnis als Ganzes zu neuen Konditionen fort, noch schliesst er mit einem neuen Gläubiger einen Darlehensvertrag ab. In einem solchen Fall fehlt die nötige Beziehung zur Darlehensschuld die einen Abzug als Schuldzins rechtfertigen würde. ${ }^{8}$

11 Das Bundesgericht kommt daher zum Schluss, dass die Vorinstanz mit der Bestätigung des Veranlagungsentscheids, die Vorfälligkeitsentschädigung bei der Einkommenssteuer nicht zum Abzug zuzulassen, kein Bundesrecht verletzt hat. Die Beschwerde wird deshalb abgewiesen.

\footnotetext{
BGE 2C_1165/2014 vom 3. April 2017, E. 2.1. BGE 2C_1165/2014 vom 3. April 2017, E. 2.2. Im Urteil wird irrtümlicherweise auf Art. $60 \mathrm{ff}$. OR verwiesen.

5 BGE 2C_1165/2014 vom 3. April 2017, E. 2.3, mit Hinweis auf RDAF 2012 II S. 92, mit ausführlichen Bemerkungen von Raphael Gani.
}

6 BGE 2C_1165/2014 vom 3. April 2017, E. 2.3 i.f.

7 BGE 2C_1165/2014 vom 3. April 2017, E. 2.3 i.f., unter Hinweis auf BGE 2C_1148/2015 vom 3 . April 2017, E. 5.3.1.

8 BGE 2C_1165/2014 vom 3. April 2017, E. 2.3. und 3 . 
III. BGE 2C 1148/2015 vom 3. April $\mathbf{2 0 1 7}$

\section{Sachverhalt}

12 Ein Geschäftshaus im Kanton Zürich sollte ohne hypothekarische Belastung verkauft werden. Die Verkäufer lösten die bestehenden Festhypotheken daher gegen Bezahlung einer Vorfälligkeitsentschädigung auf. Bei der Einkommenssteuer der Verkäufer wurde die Entschädigung zum Abzug zugelassen und in der Folge ein steuerbares Einkommen von CHF o.- veranlagt. Der verbliebene Schuldzinsenüberschuss wurde dann bei der Grundstückgewinnsteuer geltend gemacht.

13 Die Kommission für Grundsteuern der Stadt Zürich liess die entrichtete Vorfälligkeitsentschädigung bei der Ermittlung des Grundstückgewinns nicht zum Abzug zu. Die dagegen gerichtete Einsprache blieb ebenso erfolglos wie die nachfolgende Beschwerde an das Verwaltungsgericht des Kantons Zürich.

14 Daraufhin beantragten die Verkäufer mit Beschwerde in öffentlich-rechtlichen Angelegenheiten vor Bundesgericht, das verwaltungsgerichtliche Urteil aufzuheben. Die Vorfälligkeitsentschädigung sei bei der Bemessung des Grundstückgewinns im Umfang des Schuldzinsenüberschusses gewinnmindernd zu berücksichtigen.

\section{Erwägungen}

15 Für die Beurteilung der Frage der Abzugsfähigkeit von Vorfälligkeitsentschädigungen bei der Grundstückgewinnsteuer knüpft das Bundesgericht zunächst bei der Steuerharmonisierung an.
Das StHG9 bestimmt die von den Kantonen zu erhebenden direkten Steuern, legt die Grundsätze fest, nach denen die kantonale Gesetzgebung zu gestalten ist, ${ }^{10}$ und bezweckt eine Harmonisierung der direkten Steuern sowohl zwischen den Kantonen (horizontale Harmonisierung) als auch zwischen Bund und Kantonen (vertikale Harmonisierung). ${ }^{11}$

16 Art. 12 StHG schreibt den Kantonen die Erhebung einer Grundstückgewinnsteuer vor, belässt ihnen aber grosse Freiheiten in deren Ausgestaltung. Insbesondere umschreibt das StHG nicht, welche Kosten als Aufwendungen anrechenbar sind und sich dadurch mindernd auf den Gewinn auswirken.

17 Der Freiraum der Kantone ist jedoch nicht unbeschränkt. Aufgrund der angestrebten Harmonisierung ist eine identische Auslegung der Rechtsbegriffe insbesondere dann geboten, wenn bundessteuergesetzliche und harmonisierungsrechtliche Regelungen dem Sinne nach übereinstimmen. ${ }^{12}$ Die Grundstückgewinn- sowie die Einkommens- und Gewinnsteuer sind eng miteinander verbunden und verlangen nach einer genauen Abgrenzung. ${ }^{13}$ Ebenso ist eine doppelte Berücksichtigung der Aufwendungen ausgeschlossen. Die Abgrenzung hat darum auch sicherzustellen, dass Auslagen, welche bei der Grundstückgewinnsteuer

9 Bundesgesetz vom 14. Dezember 1990 über die Harmonisierung der direkten Steuern der Kantone und Gemeinden (StHG; SR 642.14).

10 Vgl. Art. 1 Abs. 1 StHG.

11 Reich Markus/Beusch Michael, in: Martin Zweifel/Michael Beusch (Hrsg.), Kommentar zum Schweizerischen Steuerrecht, I/1, Bundesgesetz über die Harmonisierung der direkten Steuern der Kantone und Gemeinden (StHG), Basel 2017, Vor Art. 1/2, N $29 \mathrm{ff}$.

12 BGE 2C_1148/2015 vom 3. April 2017, E. 4.1.

13 BGE 2C_1148/2015 vom 3. April 2017, E. 4.1.1. 
berücksichtigt wurden, nicht auch noch im Rahmen der Einkommenssteuer abgezogen werden können (und umgekehrt). ${ }^{14}$

Die Einhaltung zwingender Vorgaben der Harmonisierung wird vom Bundesgericht mit voller Kognition geprüft. Das gilt insbesondere für die in Art. 12 StHG erwähnten Anlagekosten in Abgrenzung zu den bei der Einkommenssteuer abzugsfähigen Unterhaltskosten. ${ }^{15}$

19 Der Begriff der "Anlagekosten» gemäss StHG bzw. § 219 Abs. 1 StG/ZH ${ }^{16}$ wurde daher präzisiert. Verlangt wird das Vorliegen wertvermehrender Aufwendungen, d.h. solcher, welche nicht bloss der Werterhaltung dienen. ${ }^{17}$ Erforderlich ist zudem, dass die Kosten vom Veräusserer effektiv aufgewendet wurden («Prinzip der effektiven Kostenanrechnung»). Die Wertvermehrungen können dabei nicht bloss körperlicher, sondern auch rechtlicher Natur sein, etwa indem ein beschränktes dingliches Recht begründet oder eine solche Last abgelöst wird.18 Auch der Wegfall eines obligatorischen Rechts kann eine Wertvermehrung des Grundstücks bewirken. ${ }^{19}$

Weiter wird darauf abgestellt, ob die Kosten im Hinblick auf die Veräusserung des Grundstücks getätigt worden sind. ${ }^{20}$ Das ergibt sich nicht nur aus den zwingenden Vorgaben der vertikalen und horizontalen Harmonisierung, sondern auch aus

17 BGE 2C_1148/2015 vom 3. April 2017, E. 4.2.1.

18 BGE 2C_1148/2015 vom 3. April 2017, E. 4.2.2 und 4.2.3.

19 BGE 2C_1148/2015 vom 3. April 2017, E. 4.2.3.

20 BGE 2C_1148/2015 vom 3. April 2017, E. 4.3. dem Grundsatz der Besteuerung nach der wirtschaftlichen Leistungsfähigkeit gemäss Art. 127 Abs. 2 BV. ${ }^{21}$

21 Anschliessend wird Bezug genommen auf das gleichentags ergangene "Neuenburger» Urteil BGer 2C_1165/2014 bzw. 2C_1166/2014, ${ }^{22}$ gemäss welchem verschiedene Sachverhaltshypothesen unterschieden werden müssen.

22 Eine Behandlung der Vorfälligkeitsentschädigung als Schuldzins ist gerechtfertigt, wenn das bestehende Darlehensverhältnisses nur hinsichtlich der Konditionen - insbesondere dem Zinssatz angepasst wird, oder beim Wechsel in ein anderes Hypothekarmodell bei demselben Kreditgeber (erste Sachverhaltshypothese). Bei einer solchen Umschuldung wird das vorherige Schuldverhältnis nicht beendet, sondern nur verändert. Werden lediglich die Modalitäten angepasst, so ist die für eine Qualifikation als Schuldzins notwendige Verbindung zwischen der Darlehenshingabe und der dafür ausgerichteten Vergütung auch in Bezug auf die Vorfälligkeitsleistung gegeben und sie kann vom Einkommen abgezogen werden. ${ }^{23}$

23 Die zweite Sachverhaltshypothese betrifft jene Fälle, in denen die bestehende Hypothek durch eine neue Hypothek bei einem anderen Kreditgeber abgelöst wird. In einem solchen Fall kann nicht gesagt werden, dass die Entschädigung innerhalb des weiter bestehenden, glei-

21 BGE 2C_1148/2015 vom 3. April 2017, E. 4.4 und 4.4.1.

22 Vgl. Das Richtergremium im «Zürcher» sowie im «Neuenburger» Fall setzte sich - mit Ausnahme der Gerichtsschreiber - aus den gleichen Mitgliedern zusammen.

23 BGE 2C_1148/2015 vom 3. April 2017, E. 5.3.1. 
chen Schuldverhältnisses erfolgt sei. Eine Gleichstellung mit Schuldzinsen rechtfertigt sich diesfalls nicht. ${ }^{24}$

24 Die dritte - im besagten Fall zu beurteilende - Sachverhaltshypothese betrifft Vorfälligkeitsentschädigungen, die im Hinblick auf den Verkauf bezahlt werden. Weil das Darlehensverhältnis beendet wird, kommt eine Gleichstellung mit Schuldzinsen ebenfalls nicht in Frage. Stattdessen stellt sich die Frage, ob die Entschädigung grundstückgewinnsteuermindernd berücksichtigt werden kann. ${ }^{25}$

Im konkreten Fall sind für die Vorfälligkeitsentschädigung sämtliche Voraussetzungen für die Annahme von «Anlagekosten» gemäss Art. 12 Abs. 1 StHG bzw. $\S 219$ Abs. 1 StG/ZH erfüllt. Die Auflösung der Festhypothek erfolgte in Hinblick auf die geplante Veräusserung des Grundstücks. ${ }^{26}$ Zudem geht es um effektive und durch die Veräusserer getätigte Aufwendungen. ${ }^{27}$ Das Vorliegen einer Wertvermehrung wird ebenfalls bejaht, da nur der Wegfall der Hypotheken es ermöglichte, die Liegenschaft zum vereinbarten Preis zu verkaufen. Dies ist mit einer rechtlichen Wertvermehrung vergleichbar, insbesondere einer solchen mittels Aufhebung schuldrechtlicher Belastungen. ${ }^{28}$

Das Bundesgericht kommt zum Schluss, dass die geltend gemachte Vorfälligkeitsentschädigung, soweit nicht bereits bei der Einkommenssteuer in Abzug gebracht, bei der der Grundstückgewinnsteuer zu berücksichtigen ist. Die Be- schwerde wird daher gutgeheissen, das angefochtene Urteil aufgehoben und die Sache im Sinne der Erwägungen an die Veranlagungsbehörde zurückgewiesen.

\section{Fazit der beiden Entscheide}

27 Das Bundesgericht unterscheidet bei Vorfälligkeitsentschädigungen drei Fälle:

- Bei der Ablösung einer Festhypothek und Umwandlung in ein anderes Hypothekarmodell bei demselben Kreditgeber liegt ein weiter bestehendes, nur hinsichtlich der Konditionen umgestaltetes Darlehensverhältnis vor. Wirtschaftlich betrachtet steht die Vorfälligkeitsentschädigung diesfalls den Schuldzinsen nahe und kann darum vom Einkommen abgezogen werden.

- Bei der Ablösung einer Festhypothek durch eine neue Hypothek bei einem andern Kreditgeber rechtfertigt sich eine Gleichstellung mit Schuldzinsen nicht. Die Vorfälligkeitsentschädigung ist in einem solchen Fall als Schadenersatz oder Wandelpön ${ }^{29} \mathrm{zu}$ qualifizieren und kann nicht vom Einkommen abgezogen werden.

- Bei der Ablösung einer Festhypothek im Hinblick auf die Veräusserung kann die Vorfälligkeitsentschädigung bei der Grundstückgewinnsteuer als (rechtliche) Wertvermehrung in Abzug gebracht werden.
BGE 2C_1148/2015 vom 3. April 2017, E. 5·3.2.
BGE 2C_1148/2015 vom 3. April 2017, E. 5·3·3.
BGE 2C_1148/2015 vom 3. April 2017, E. 5.5.1.
BGE 2C_1148/2015 vom 3. April 2017, E. 5·5.2.
BGE 2C_1148/2015 vom 3. April 2017, E. 5.5·3.
29 Art. 160 Abs. 3 OR. 


\section{Anmerkungen}

\section{Vorbemerkungen}

28 Die bis anhin unterschiedlichen kantonalen Praktiken und Lehrmeinungen machen deutlich, dass die Rechtslage im hier interessierenden Fragenkomplex nicht ohne weiteres klar ist. Die bundesgerichtliche Beurteilung ist daher zu begrüssen und bringt eine gewisse Vereinheitlichung sowie Rechtssicherheit und Planbarkeit mit sich. Angesichts der Erwägungen stellen sich dem Autor jedoch verschiedene Fragen, denen im Folgenden nachgegangen wird.

\section{Bisherige kantonale Praxis}

29 In den Kantonen Basel-Landschaft 30 , Basel-Stadt31, Bern32, Luzern33, St. Gallen 34 , Thurgau 35 und Zürich ${ }^{6}$ konnte die Vorfälligkeitsentschädigung bis anhin grundsätzlich beim Einkommen in Abzug gebracht werden, nicht dagegen im Kanton Neuenburg. 37

30 Entscheid des Steuergerichts des Kantons BaselLandschaft Nr. 131 vom 26. November 2004.

31 Urteil des Appellationsgerichts des Kantons Basel-Stadt als Verwaltungsgericht vom 16. Juni 2008; Merkblatt betreffend den Abzug von Liegenschaftskosten, Ausgabe vom 31. Dezember 2016, S. 19.

32 TaxInfo, Schuldzinsen, Kanton Bern, Fassung vom 07. April 2016.

33 Vgl. Luzerner Steuerbuch, Band 1, Weisungen StG: Einkommenssteuer, § 40 Nr. 1 vom 01. Januar 2016 und Steuerbulletin der Steuerverwaltung des Kantons Luzern, 03/2006, S. 4; anders noch LGVE 1998 II Nr. 30,worauf das Bundesgericht für den Kanton Luzern wohl irrtümlich weiterhin verweist.

34 St. Galler Steuerbuch, StB 45 Nr. 5, Ausgabe vom o1. Januar 2016, Vorfälligkeitsentschädigung.

35 Steuerpraxis Thurgau, StP 34 Nr. 9, Festhypothek, Portfolio-Hypothek, LIBOR-Hypothek, Ausgabe vor 26. Juni 2017.

36 Richner Felix/Frei Walter/Kaufmann Stefan/ Meuter Hans Ulrich, Kommentar zum Zürcher Steuergesetz, Zürich 2013, § 31 N 15.

37 Arrêt du Tribunal cantonal du canton de Neuchâtel, 17. August 2011, CDP.2011.99, in RDAF II 2012, S. 92.
3 B Bemerkenswert ist, dass einzelne frühere kantonale Praktiken, 38 welche den Gläubigerwechsel als entscheidenden Umstand ansahen, bereits wieder aufgegeben wurden bevor nun das Bundesgericht darauf abstellt. Im Fall des Steuergerichts des Kantons Basel-Landschaft wurde die Abkehr vom Kriterium des Gläubigerwechsels insbesondere damit begründet, dass das Abstellen auf die Umfinanzierung beim gleichen Kreditinstitut das Rechtsgleichheitsgebot verletze, dass der Grundsatz der Besteuerung auf Grund der wirtschaftlichen Leistungsfähigkeit den Abzug verlange und dass es sich dabei um Gewinnungskosten im Sinne der Generalklausel von Art. 9 Abs. 1 StHG handle. Bedauerlicherweise gehen beide Bundesgerichtsentscheide nicht auf diese Argumentationen ein.

\section{Relevanter Gläubigerwechsel?}

31 Das Bundesgericht macht die einkommensteuerliche Abzugsfähigkeit von der Tatsache abhängig, ob im Zusammenhang mit der Vorfälligkeitsentschädigung eine Umfinanzierung beim gleichen Gläubiger erfolgt. In der Begründung des «Zürcher» Urteils39 wird ausgeführt, dass bei einem Gläubigerwechsel eine Gleichstellung mit Schuldzinsen nicht gerechtfertigt sei. Verwiesen wird dabei auf das «Neuenburger» Urteil.40 Dieses enthält besagte Aussage jedoch nicht in dieser Form sondern hält lediglich fest, dass bei einer Umschuldung bei demselben Gläubiger die Vorfälligkeitsentschä-

$3 8 \longdiv { \text { Beispielsweise Basel-Landschaft (Praxisänderung } }$ gemäss Entscheid des Steuergerichts des Kantons Basel-Landschaft Nr. 131 vom 26. November 2004) und Luzern (Steuerbulletin der Steuerverwaltung des Kantons Luzern, 03/2006, S. 4).

39 BGE 2C_1148/2015 vom 3. April 2017, E. 5.3.2.

40 BGE 2C_1165/2014 vom 3. April 2017, E. 2.3 und E. 3 . 
digung eher einem Entgelt als einem Schadenersatz entspreche. Ob der Umkehrschluss gezogen werden kann, wird gerade nicht gesagt. Das Gericht verweist in seinen Erwägungen auf die von Raphael Gani ${ }^{41}$ vertretene differenzierte Auffassung und erklärt, dass dieser gefolgt werden müsse. Gani hält bezüglich des Gläubigerwechsels jedoch fest, «qu'il n'apparait pas déterminant que le nouveau contrat soit conclu avec le même créancier ou avec un autre, cette donnée ne modifiant en rien la nature de l'indemnité». Seine diesbezügliche Auffassung deckt sich also gerade nicht mit jener des Bundesgerichts. Der Widerspruch wird in der Folge leider nicht thematisiert, so dass unklar bleibt, ob eine bewusste Abkehr erfolgt ist. Ebenso wird die Frage nicht explizit behandelt, ob das Abstellen auf den Gläubigerwechsel unter dem Gesichtspunkt der Rechtsgleichheit haltbar ist. ${ }^{2}$ Nach Ansicht des Autors ist dies zu verneinen. 43

\section{Verkaufsbedingte Anlagekosten?}

32 Das Bundesgericht prüft des Weiteren die grundstückgewinnsteuerrelevante Qualifikation der Vorfälligkeitsentschädigung als «Anlagekosten» gemäss Art. 12 Abs. 1 StHG. 44 Da vorliegendenfalls die Hypothek unmittelbar vor dem Verkauf aufgelöst und nicht durch eine neue Hypothek ersetzt wurde liegt gemäss Bundesgericht eine rechtliche Wertvermehrung vor. Die Kosten können darum bei der Grundstückgewinnsteuer

41 RDAF 2012 II S. 92.

42 Vgl. dazu etwa den Entscheid des Steuergerichts des Kantons Basel-Landschaft Nr. 131 vom 26. November 2004.

43 Vgl. nachfolgend Ziffer 6. Rechtsnatur der Vorfälligkeitsentschädigung i.f.

44 BGE 2C_1148/2015 vom 3. April 2017, E. 5.5. abgezogen werden. 45

33 In den Erwägungen werden also «Anlagekosten» und «Gewinnungskosten» kombiniert, was die dogmatische Einordnung der Begründung erschwert. Die vom Bundesgericht verlangte untrennbare Verbindung einer Auslage mit dem Erwerb bzw. - wie im hier interessierenden Fall - mit der Veräusserung ist lediglich unter dem Aspekt der Gewinnungskosten ${ }^{46}$ relevant. Nach Ansicht des Autors kann es dagegen für die Abzugsfähigkeit als Anlagekosten nicht darauf ankommen, ob die Aufwendung mit der Veräusserung zusammenhängt. Baukosten - um einen Paradefall von Anlagekosten zu nennen - sind bei der Grundstückgewinnsteuer zweifellos gewinnmindernd $\mathrm{zu}$ berücksichtigen, obschon von ihnen gerade nicht gesagt werden kann, dass sie im Zusammenhang mit dem Erwerb oder der Veräusserung stehen. Wenn mit dem Vorliegen einer rechtlichen Wertvermehrung argumentiert wird, so muss dies auch für jene Grundstücke gelten, welche vorerst nicht verkauft werden. Bei einem späteren Verkauf würde die Entschädigung dann, wie z.B. die Baukosten auch, grundstückgewinnsteuerlich zum Abzug gelangen.

\section{Rechtliche Wertvermehrung?}

34 Eine Wertvermehrung bezieht sich stets auf ein konkretes Objekt. Beim Hypothekarkredit handelt es sich um eine persönliche Schuldpflicht, die vorerst ohne Grundstückbezug ist. Erst durch die Sicherstellung mittels Grundpfandrecht wird ein solcher Objektbezug hergestellt. Werden Schuldbriefe auf verschiedenen

$45 \overline{\text { BGE 2C_1148/2015 vom 3. April 2017, E. } 4.3 \text { und }}$ 5.5.1.

46 Vgl. die Generalklausel von Art. 9 Abs. 1 StHG. 
Grundstücken zur Besicherung einer Gesamtfinanzierung verwendet, so ist eine Zuordnung der Vorfälligkeitsentschädigung zu nur einem der grundpfandbelasteten Grundstücke - nämlich zu jenem, welches verkauft wird - nicht möglich. Es liesse sich sachlich nicht rechtfertigen, warum das Verkaufsobjekt anders behandelt werden sollte als die anderen pfandbelasteten Grundstücke. Der Grundstückbezug fehlt zudem ganz, wenn an Stelle eines Schuldbriefs beispielsweise eine Lebensversicherung oder börsengängige Wertpapiere verpfändet werden. Wird ein derart finanziertes Grundstück verkauft und fällt dabei eine Vorfälligkeitsentschädigung an, so dürfte mangels eines Objektbezugs eine rechtliche Wertvermehrung des Grundstücks nicht zur Diskussion stehen. Der Autor geht darum beim zu beurteilenden Sachverhalt nicht vom Vorliegen einer (rechtlichen) Wertvermehrung aus.

\section{Rechtsnatur der Vorfälligkeitsent- schädigung}

35 Im Zusammenhang mit der Frage der Abzugsfähigkeit wird regelmässig thematisiert, wie Vorfälligkeitsentschädigungen rechtlich $\mathrm{zu}$ qualifizieren sind bzw. ob einer streng rechtlichen oder einer eher wirtschaftlichen Betrachtungsweise gefolgt wird.

36 Es liegt auf der Hand, dass die Vorfälligkeitsentschädigung kein Schuldzins im engen Sinn ist. Nach Lehre und Rechtsprechung handelt es sich entweder um eine Konventionalstrafe im Sinne von Art. 160 Abs. 1 OR47 oder um vertrag-

47 Bundesgesetz vom 30. März 1911 betreffend die Ergänzung des Schweizerischen Zivilgesetzbuches (Fünfter Teil: Obligationenrecht) (OR; SR 220). lichen Schadenersatz im Sinne von Art. 97 ff. OR.

37 Nach Ansicht des Autors ist davon auszugehen, dass die Darlehensschuld angesichts der vereinbarten festen Laufzeit vor Ablauf gar nicht erfüllbar ist. ${ }^{8}$ Der Schuldner kann die nicht erfüllbare Schuld darum vorzeitig gar nicht tilgen und kann deshalb dadurch auch keine Vertragsverletzung begehen.

38 Wenn im Darlehensvertrag die vorzeitige Ablösung vorgesehen ist oder wenn bei Bedarf nachträglich eine solche vereinbart wird (was nötig wäre, wenn die Schuld als noch nicht erfüllbar angesehen wird), dann dürfte eine Vertragsverletzung und damit ein Schadenersatz ausser Betracht fallen. Die Entschädigung ist daher als Konventionalstrafe, und zwar in Form der Wandelpön im Sinne von Art. 160 Abs. 3 OR zu qualifizieren. 49

39 Im Ergebnis ist es jedoch wirtschaftlich vergleichbar, ob ein Schuldzins vorliegt oder eine Ablöseentschädigung, welche in Abhängigkeit von Kapitalbetrag, Dauer und dem Zinsmargenverlust des Gläubigers festgesetzt wird.5o Der Unterschied liegt letztlich primär beim Zeitpunkt der Zahlung, wobei auch beim Schuldzins im engen Sinn eine vorschüssige Bezahlung der Abzugsfähigkeit nicht entgegensteht. $5^{1}$

48 Art. 81 Abs. 1 OR.

49 Stadlin Markus W., Der Liegenschaftsverkauf mit vorzeitiger Auflösung der Festhypothek: Überlegungen zur Rechtsnatur der Vorfälligkeitsentschädigung und zu den steuerlichen Folgen, BJM 2017 S. 1.

50 Auf die Frage der Abzugsfähigkeit von Gebühren oder Entschädigungen, die nicht in Abhängigkeit der genannten Kriterien festgelegt werden wird in diesem Beitrag nicht eingegangen.

51 Agner Peter/Jung Beat/Steinmann Gotthard, 
40 Gemäss Bundesgericht ist der Begriff «Schuldzins» wirtschaftlich $\mathrm{zu}$ betrachten. ${ }^{2}$ Zudem hat die Besteuerung nach der wirtschaftlichen Leistungsfähigkeit $\mathrm{zu}$ erfolgen. Es ist darum nach Ansicht des Autors nicht sachgerecht, für die Frage, ob ein Steuerabzug erfolgen könne auf die formelle Beibehaltung des Gläubigers abzustellen, insbesondere da auch in diesem Fall das konkrete Darlehensverhältnis endet.

\section{Besteuerung nach der wirtschaftli- chen Leistungsfähigkeit}

41 Beide Urteile erwähnen den Grundsatz der Besteuerung nach der wirtschaftlichen Leistungsfähigkeit (Art. 127 Abs. 2 BV) bloss am Rande, 53 obschon eine vertieftere Auseinandersetzung mit dem Thema unter mehreren Aspekten interessant gewesen wäre.

42 Die Grundstückgewinnsteuer ist als Objektsteuer ausgestaltet.54 Zum diesem Umstand, der den genannten verfassungsmässigen Grundsatz ohnehin schon strapaziert, kommt hinzu, dass die neue Praxis zu einer Verschärfung der Problematik führt.

43 Bei einem Abzug der Vorfälligkeitsentschädigung auf Stufe des steuerbaren Gewinns führt regelmässig eine lange Besitzesdauer dazu, dass die Entschädigung durch den Besitzesdauerabzug «verwässert» wird.55 Dies wiederum

Kommentar zum Gesetz über die direkte Bundessteuer, Zürich 1995, Art. 33, N 2.

52 BGE 2C_1165/2014 vom 3. April 2017, E. 2.1.

53 BGE 2C_1165/2014 vom 3. April 2017, E. 2.4, i.f., BGE 2C_1148/2015 vom 3. April 2017, E. 2.3.2, 4.4.1 und 4.4.2.

54 BGE 2C_1148/2015 vom 3. April 2017, E. 2.3.2.

55 Im Kanton Bern etwa wird der Rohgewinn um bis zu 70\% gekürzt, so dass die Ablösekosten u.U. steht der Ratio von Art. 12 Abs. 5 StHG entgegen, kurzfristig realisierte Grundstückgewinne stärker zu besteuern.

44 Noch stossender ist das Ergebnis, wenn beim Verkauf ein Verlust erlitten wird. Dann nämlich kommt die Vorfälligkeitsentschädigung steuerlich überhaupt nicht zum Tragen.

45 Die bisher vorherrschende Lehrmeinung und kantonale Praxis der Abzugsfähigkeit im Rahmen der Einkommenssteuer hatte dagegen den Vorteil, dass der Abzug im Regelfall wirtschaftlich auch wirklich zum Tragen kam. Dem Abzug stand nämlich zumeist ein Einkommen (typischerweise als Mietertrag oder Eigenmietwert) gegenüber. Zudem konnten da hier eben gerade keine Objektbetrachtung erfolgt - weitere Einkommen zur Kompensation herangezogen werden. Im Licht der Idee der Besteuerung nach der wirtschaftlichen Leistungsfähigkeit war dies zu begrüssen, obschon die zeitliche Vorverlegung an sich gegen das Periodizitätsprinzip verstiess.

46 Nach Ansicht des Autors verlangt die neue Praxis nach einer Flexibilisierung hinsichtlich der Objektbetrachtung bei der Grundstückgewinnsteuer. ${ }^{5}$ Aus ähnlichen Überlegungen ist auch eine Verrechnung von Grundstückgewinnen mit Verlusten, insbesondere auch mit Grundstückverlusten aus anderen Steuerjahren zu begrüssen. 57

nur noch $\mathrm{zu} 30 \%$ in die Steuerberechnung einfliessen obschon sie erst unmittelbar vor Verkauf angefallen sind.

56 In diesem Sinne auch die jüngeren Entwicklungen bezüglich Ausscheidungsverluste, vgl. zum Ganzen: Suter Claudia, Innerkantonale Verlustverrechnung - gerechtfertigter Sonderfall?, zsis aktuell, 1/2012, S. $4 \mathrm{ff}$.

57 Vgl. Art. 143 des Steuergesetzes vom 21. Mai 


\section{Praktische Herausforderungen}

47 Die neue Rechtsprechung mit einer nach dem Auflösungsgrund differenzierten Abzugsfähigkeit der Vorfälligkeitsentschädigung bringt für die Veranlagungsbehörden diverse praktische Herausforderungen mit sich. Exemplarisch wird hier auf drei Punkte kurz eingegangen.

48 Vordringlich wird sich die Praxis mit der Frage auseinandersetzen müssen, wie mit der neuen Rechtsprechung in laufenden Verfahren umzugehen ist. Im Vertrauen auf die fortgesetzte Anwendung der bisherigen Praxis (Abzug bei der Einkommenssteuer) haben die Steuerpflichtigen bei der Grundstückgewinnsteuererklärung jeweils auf die Geltendmachung des Abzugs für die Vorfälligkeitsentschädigung verzichtet. Die Grundstückgewinnsteuererklärung ist folglich ohne den Abzug erfolgt. Im Rahmen der Veranlagung der Einkommenssteuer wird nun der Abzug geltend gemacht, müsste angesichts der neuen Rechtsprechung nun aber abgelehnt werden. Die Aufwendung für die Vorfälligkeitsentschädigung würde demnach steuerlich ganz ins Leere fallen, was nach dem Grundsatz der Besteuerung nach der wirtschaftlichen Leistungsfähigkeit stossend wäre. Einzelne Kantone haben bereits Lösungen aufgezeigt, sei es als Korrektur bei der (bereits rechtskräftig veranlagten) Grundstückgewinnsteuer, $5^{8}$ sei es als weiterhin zugelassenen Abzug bei der Einkommenssteuer.59

2000 des Kantons Bern (BSG 661.11).

58 So der Lösungsansatz im Kanton Luzern, vgl. Newsletter Steuern Luzern vom 24.Mai 2017.

59 So der Lösungsansatz im Kanton Bern, vgl. Taxinfo Schuldzinsen vom 13.Juli 2017. Dieser Lösung ist der Verzug zu geben da die Steuerpflichtigen regelmässig im Vertrauen in die Fortführung der Praxis Dispositionen vornehmen.
49 Zudem wird die Veranlagung angesichts der neuen, differenzierten Praxis sowohl für den Steuerpflichtigen wie auch für die Behörde aufwändiger weil die näheren Umstände um die Vorfälligkeitsentschädigung genau untersucht und gegebenenfalls nachgewiesen werden müssen.

50 Ausserdem wird die neue Praxis zu heiklen Abgrenzungsfragen führen, die beim bisherigen einheitlichen Abzug als Schuldzins vermieden werden konnten. Weil im konkreten Einzelfall der Abzug der Entschädigung bei der Einkommenssteuer wesentlich vorteilhafter sein kann als bei der Grundstückgewinnsteuer (etwa bei ausserordentlichen Einnahmen, einer langen Besitzesdauer oder einem Verkauf mit Verlust), ist es im Rahmen einer Steueroptimierung naheliegend, die bestehende Festhypothek in einem ersten Schritt durch eine Finanzierung mit unbefristeter Laufzeit beim bisherigen Gläubiger abzulösen. Beim Verkauf ist dann die Ablösung dieser neuen Hypothek ohne Kostenfolge möglich. Die Veranlagungspraxis wird zeigen müssen, wie lange vor dem Verkauf eine so gestaltete, kurzfristige Umfinanzierung mindestens erfolgen muss, damit ein Abzug der Vorfälligkeitsentschädigung als Schuldzins anerkannt werden kann. Praktische Aspekte legen nahe, dass diese Frist bis zum Ende der Veranlagungsperiode dauert, in welcher die Entschädigung in Abzug gebracht wurde.

\section{Schlussbetrachtung}

51 Die neue Praxis des Bundesgerichts, man mag sie als sachlich überzeugend erachten oder nicht, führt für wirtschaftlich ziemlich ähnliche Lebenssachverhalte zu sehr unterschiedlichen Steuerfolgen. Je 
nachdem, ob die Ablösung der Hypothek im Hinblick auf einen Verkauf erfolgt, oder ob lediglich eine Umfinanzierung (ohne oder mit Gläubigerwechsel) erfolgt, reicht das Spektrum vom Abzug bei der Grundstückgewinnsteuer, über den Abzug bei der Einkommenssteuer, bis hin zur Verweigerung eines steuerlichen Abzugs.

52 Diese Differenzierung mag dogmatisch zu begründen sein. Man kann sich aber durchaus darüber streiten, ob die neue Praxis deshalb der bisher vorherrschenden, einheitlichen und darum ausgesprochen praktikablen Lösung vorzuziehen ist. 